\title{
Protective effects of andrographolide against cerebral ischemia-reperfusion injury in mice
}

\author{
YAN LI $^{1,2}$, LI-LING XIANG ${ }^{2}$, JIN-XIN MIAO $^{2}$, MING-SAN MIAO $^{2}$ and CAN WANG ${ }^{2}$ \\ ${ }^{1}$ College of Traditional Chinese Medicine; ${ }^{2}$ School of Pharmacy, \\ Henan University of Chinese Medicine, Zhengzhou, Henan 450046, P.R. China
}

Received June 8, 2020; Accepted January 8, 2021

DOI: $10.3892 / \mathrm{ijmm} .2021 .5019$

\begin{abstract}
Ischemic stroke is one of the most common causes of mortality worldwide and is a primary cause of disability and mortality in adults. There is an unmet need for drugs that can effectively treat ischemic stroke. Hence, the present study explored the neuroprotective effects of andrographolide (Andro) in a mouse model of bilateral common carotid artery occlusion, and systematically evaluated the potential mechanisms underlying its effects. The effects of Andro on mouse brain tissue following cerebral ischemia-reperfusion injury (CIRI) were evaluated by histopathological (H\&E and Nissl) and immunofluorescence [ glial fibrillary acidic protein (GFAP) and neuronal nuclei (NeuN)] staining. A traditional Chinese medicine-based network pharmacology method was performed to establish and analyze compound-target-disease and function-pathway networks in order to elucidate the possible mechanisms responsible for the protective role of Andrographis paniculata in CIRI. In addition, western blot analysis and RT-qPCR was performed to evaluate the expression and activation of
\end{abstract}

Correspondence to: Professor Ming-San Miao or Dr Jin-Xin Miao, School of Pharmacy, Henan University of Chinese Medicine, 156 Jinshui East Road, Zhengzhou, Henan 450046, P.R. China

E-mail: miaomingsan@163.com

E-mail: jinxin.miao@yahoo.com

Abbreviations: Andro, andrographolide; BDNF, brain-derived neurotrophic factor; $\mathrm{CCH}$, chronic cerebral hypoperfusion; CIRI, cerebral ischemia-reperfusion injury; DAPI, 4',6-diamidino-2-phenylindole; DL, drug-likeness; ELISA, enzyme-linked immunosorbent assay; GFAP, glial fibrillary acidic protein; GO, gene ontology; H\&E, hematoxylin and eosin; IL-1 $\beta$, interleukin $1 \beta$; IL-6, interleukin 6; KEGG, Kyoto Encyclopedia of Genes and Genomes; MCAO, middle cerebral artery occlusion; NeuN, neuronal nuclei; OB, oral bioavailability; PI3K, phosphoinositide 3-kinase; PVDF, polyvinylidene fluoride; RT-qPCR, reverse transcription-quantitative polymerase chain reaction; TBST, Tris-buffered saline/Tween-20; TNF- $\alpha$, tumor necrosis factor- $\alpha$

Key words: andrographolide, cerebral ischemia-reperfusion injury, TCM-based network pharmacology, PI3K/Akt signaling proteins predicted to be involved in this mechanism. The amelioration of histopathological alterations was observed in mice pre-treated with Andro. Immunofluorescence staining revealed that Andro decreased the expression of GFAP and increased the expression of NeuN, and significantly decreased the levels of pro-inflammatory cytokines $(I L-1 \beta, I L-6$ and $T N F-\alpha$ ). Network pharmacology analysis revealed that neuroinflammatory response and apoptosis were associated with the effects of Andrographis paniculata on CIRI. Western blot analysis revealed that the mice pre-treated with Andro exhibited an upregulated protein expression of tropomyosin receptor kinase B (TrkB), p-PI3K and p-Akt, as well as a decrease in the expression of GFAP and an increase in the expression of NeuN. In addition, the data of RT-qPCR indicated that the mice pre-treated with Andro exhibited a significantly decreased expression of encoding $I L-1 \beta$ mRNA, $I L-6$ mRNA and $T N F-\alpha$ mRNA in the brain compared to the untreated mice following CIRI. On the whole, the findings of the present study suggest that pre-treatment with Andro exerts a protective effect against CIRI, which may be partly related to its potential to reduce neuroinflammatory response and apoptosis in patients with stroke.

\section{Introduction}

Stroke is one of the leading causes of human mortality and long-term disability worldwide (1), and its annual incidence is gradually increasing. Approximately $80 \%$ of stroke cases involve ischemic stroke, which affects the daily life and work of patients, and places a heavy burden on family and society (2). Brain ischemia is caused by blood vessel obstruction (insufficiency) and brain damage is caused by vascular occlusion (3). The greater the decrease in cerebral blood flow, the more severe the brain damage (4). The pathophysiological mechanisms are complex and include inflammation, apoptosis, excitotoxicity and periinfarct depolarization (5). The reduction of inflammation and elimination of inflammatory genes exerts neuroprotective effects against brain damage following ischemic stroke (6). In addition, cerebral ischemia can affect the innate and adaptive immune systems, and can induce neuronal apoptosis (7). Although drugs have been developed to target inflammation, oxidative stress, apoptosis and other pathophysiological mechanisms, there remains a lack of effective neuroprotectors to prevent cerebral ischemia (8). 
Thrombolytic therapy is one of the strategies recommended for the treatment of brain ischemia; however, its use is limited by a very narrow time window and adverse effects (9). Recombinant tissue plasminogen activator, a thrombolytic agent, is the only approved thrombolytic therapy, and it must be administered within a limited time frame in order for a clinical benefit to be achieved (10). Therefore, the identification of novel treatment strategies for stroke is essential. Notably, traditional Chinese medicine (TCM) has a long history in the treatment of ischemic stroke.

Chuanxinlian is the dry, aerial part of Andrographis paniculata, and one of its main components is andrographolide (Andro). In a previous study, a rat model of permanent middle cerebral artery occlusion (pMCAO) was established to investigate the potential therapeutic effects of Andro in cerebral ischemia; it was found that after Andro was administered intraperitoneally to rats with pMCAO, the neurological behavior scores and infarction area were decreased (11). Andro may exert therapeutic effects against hippocampal neuron injury and cognitive dysfunction induced by chronic cerebral hypoperfusion $(\mathrm{CCH})$, and it also exerts a potential neuroprotective effect in cases of hippocampal neuronal damage and cognitive impairment from $\mathrm{CCH}$ due to the suppression of astrocyte activation (12). The present study aimed to further explore the effects of Andro on cerebral ischemia-reperfusion through both network analysis and in vivo experiments.

Network pharmacology is a novel drug research method that combines systems biology, multi-direction pharmacology, network analysis and computer technology. The association between drugs and disease has been investigated by combining drug component targets and disease targets from multiple perspectives $(13,14)$. In the present study, the active components and disease targets of Chuanxinlian against ischemic stroke were summarized using the database of network pharmacology, and the association between the active components of Chuanxinlian, targets and pathways was explored through multi-directional systems biology. A TCM-based network pharmacology method was used to establish and analyze compound-target-disease and function-pathway networks in order to elucidate the possible mechanisms through which Andrographis paniculata exerts its protective effects against cerebral ischemia-reperfusion injury (CIRI). The classical pharmacology of Andro was also studied in vivo to verify the results of network pharmacology. Our study aims to provide a scientific basis for the clinical application of Andro in stroke. In addition, this study provides theoretical support for subsequent experimental studies.

\section{Materials and methods}

Animals. A total of 90 male 6-week-old specific pathogen-free (SPF) Kunming mice weighing 18-22 g were obtained from Jinan Pengyue Experimental Animal Breeding Co., Ltd. (Laboratory animal certificate: SCXK 2014-0007). The animals were housed in cages with an ambient temperature of $23-25^{\circ} \mathrm{C}$ and a humidity of $35-45 \%$, under a 12-h light/dark cycle beginning at 08:00; standard mouse chow and water were provided ad libitum. The present study was performed in accordance with the recommendations of the Ministry of Science and Technology of China's Guidance for the Care and

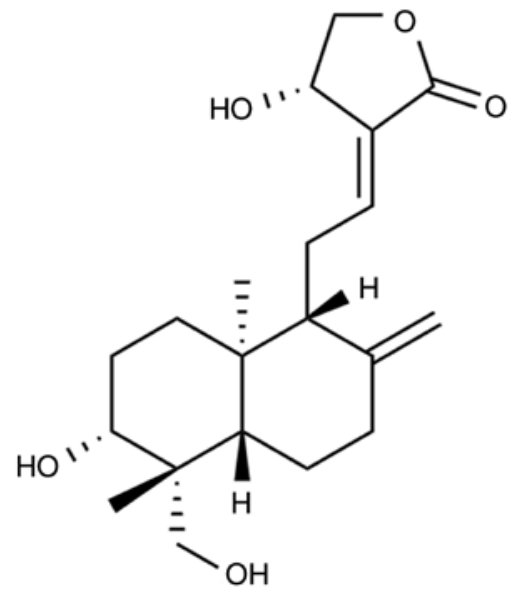

Figure 1. Chemical structure of andrographolide.

Use of Laboratory Animals. All procedures were approved by the Laboratory Animal Ethics Committee of Henan University of Chinese Medicine (the number of approval ethics code: DWLL201903026).

Drug administration. Using high-performance liquid chromatography, the purity of Andro provided by Shanghai Yuanye Bio-Technology Co., Ltd. (chemical structure presented in Fig. 1) was determined to be $98 \%$. The maximum dose of Andro used in the present study $(120 \mathrm{mg} / \mathrm{kg} ; 12 \mathrm{mg} / \mathrm{ml})$ was selected based on a previous study (15) and preliminary experiments (data not shown). A $200 \mathrm{mg} / \mathrm{ml}$ solution of Andro dissolved in dimethyl sulfoxide was prepared and diluted in distilled water to the appropriate concentration prior to use. The animals were randomly assigned to 5 groups as follows: The sham-operated group $(n=10)$, repeated CIRI group $(n=20)$ and 3 Andro groups (30, 60 and $120 \mathrm{mg} / \mathrm{kg})(\mathrm{n}=20)$. Following randomization, the corresponding drugs were administered orally, with the sham-operated group and CIRI group being treated with an equal volume of normal saline. At $1 \mathrm{~h}$ after the final administration on the 14th day, the mice in each group were subjected to repeated CIRI surgery.

Repeated CIRI in mice. Repeated CIRI was performed through bilateral common carotid artery occlusion (BCCAO) according to a previously published method (16). In brief, anesthesia was initialized with $4 \%$ isoflurane inhalation and then maintained with $1.5 \%$ isoflurane in $70 \%$ nitrous oxide $\left(\mathrm{N}_{2} \mathrm{O}\right) / 30 \%$ oxygen $\left(\mathrm{O}_{2}\right)$ using a laboratory anesthesia system (Matrix VIP 3000; Midmark Corp.). The bilateral common carotid arteries (BCCAs) were exposed, the carotid artery was lifted with 4-0 silk sutures, and the BCCAs were ligated with artery clamps for $10 \mathrm{~min}$. The artery clamps were then removed and the BCCAs were visually inspected for reperfusion. After the blood flow had been restored for $10 \mathrm{~min}$, the BCCAs were again ligated for $10 \mathrm{~min}$. The sham-operated mice received the same procedure, but without carotid artery ligation. Wounds were sutured with a 4-0 silk suture. During the surgery, the temperature was maintained at $37 \pm 0.5^{\circ} \mathrm{C}$. To ameliorate the suffering of animals observed throughout the experimental period, the animals were euthanized with $4 \%$ isoflurane inhalation followed by the dislocation of the 
Table I. Sequences of primers used for RT-qPCR.

\begin{tabular}{lll}
\hline Gene & \multicolumn{1}{c}{ Sense primer (5'-3') } & \multicolumn{1}{c}{ Antisense primer (5'-3') } \\
\hline$\beta$-actin & GTGACGTTGACATCCGTAAAGA & GTAACAGTCCGCCTAGAAGCAC \\
BDNF & GCCCATGAAAGAAGTAAACGTCC & AGTGTCAGCCAGTGATGTCGTC \\
IL-6 & TTCTTGGGACTGATGCTGGTG & GCCATTGCACAACTCTTTTCTC \\
IL-1 $\beta$ & ACAGGCTCCGAGATGAACAAC & GTGGGTGTGCCGTCTTTCAT \\
TNF- $\alpha$ & ACCCTCACACTCACAAACCA & ATAGCAAATCGGCTGACGGT
\end{tabular}

BDNF, brain-derived neurotrophic factor; IL, interleukin; TNF- $\alpha$, tumor necrosis factor $\alpha$.

cervical vertebra. Animal death was confirmed by respiratory and cardiac arrest, and no righting reflex. The brain tissues were removed $(n=3)$, then fixed in $4 \%$ paraformaldehyde and embedded in paraffin prior to hematoxylin and eosin (H\&E), Nissl and immunofluorescence staining. The other brain tissues of the mice were cryopreserved in liquid nitrogen for western blot analysis, reverse transcription-quantitative polymerase chain reaction (RT-qPCR) and biochemical detection.

$H \& E$ staining. Mouse brains were excised and fixed overnight in $4 \%$ paraformaldehyde at $4^{\circ} \mathrm{C}$, embedded in paraffin and serial $5-\mu \mathrm{m}$-thick sections were prepared. The sections were stained with H\&E (C0105, Beyotime Institute of Biotechnology) to assess brain histology. Briefly, the sections were stained with hematoxylin for $5 \mathrm{~min}$, then washed with running water for $5 \mathrm{~min}$ and differentiated in $1 \%$ acid alcohol for $10 \mathrm{sec}$ at room temperature. The sections were then stained with $1 \%$ eosin for $1 \mathrm{~min}$, then washed with running water and dehydrated in a decreasing concentration of alcohol $(70,80$, 90 and 100\%) and cleared in xylene. Finally, the sections were mounted with neutral resin and observed under a microscope (Olympus, BX63; Olympus Corporation).

Nissl staining. The brain tissue sections were stained with Nissl staining solution (C0117, Beyotime Institute of Biotechnology) for $30 \mathrm{~min}$ at $37^{\circ} \mathrm{C}$. The brain sections were then washed with 95\% ethyl alcohol and observed under a microscope (Olympus, BX63; Olympus Corporation). Large cell bodies with abundant cytoplasm and significant levels of Nissl bodies represent normal neurons. Cells with pyknosis or blurring of Nissl bodies represent damaged cells.

Immunofluorescence staining. Serial 5- $\mu \mathrm{m}$-thick paraffinembedded sections were baked at $60^{\circ} \mathrm{C}$ for $30 \mathrm{~min}$, deparaffinized with xylene and rehydrated. The sections were then submerged into the citrate antigen retrieval buffer, heated in a microwave for antigen retrieval, treated with $3 \%$ hydrogen peroxide to quench endogenous peroxidase activity and incubated with $1 \%$ bovine serum albumin to block non-specific binding. The sections were incubated with primary antibody [glial fibrillary acidic protein (GFAP), 1:500, GB12096, Wuhan Servicebio Technology Co., Ltd.; neuronal nuclei (NeuN), 1:500, GB11138, Wuhan Servicebio Technology Co., Ltd.] overnight at $4^{\circ} \mathrm{C}$. After washing, the tissue sections were treated with fluorescence-conjugated secondary antibody (G1213-100UL, Wuhan Servicebio Technology Co., Ltd.) in the dark $\left(37^{\circ} \mathrm{C}\right)$ for $2 \mathrm{~h}$. After washing with PBS, the sections were immediately examined under a fluorescence microscope (Olympus, BX63; Olympus Corporation).

Cytokine analysis. The infarct sides of brain tissues following repeated CIRI surgery were used for assessing expression brain-derived neurotrophic factor (BDNF) and pro-inflammatory cytokines. The levels of BDNF and pro-inflammatory cytokines in the supernatant of the brain homogenates or serum were detected by enzyme-linked immunosorbent assay (ELISA).

Western blot analysis. The brain tissues of the mice were removed from the liquid nitrogen and placed in a pre-cooled mortar that had been cleaned and dried at high temperature in advance. The liquid nitrogen was poured into the mortar, and the tissues were ground into a powder in the liquid nitrogen. Approximately $100 \mathrm{mg}$ of brain tissue powder were weighed and placed in a 5-ml EP tube, and $1 \mathrm{ml}$ of RIPA lysis buffer containing $1 \%$ PMSF and $1 \%$ phosphatase inhibitor was added. Extraction with a syringe was performed until the tissue was completely fragmented completely and in full contact with the lysis buffer. The lysate was then placed on ice for $30 \mathrm{~min}$. Lysates were centrifuged at 12,000 $\mathrm{x} \mathrm{g}$ for $15 \mathrm{~min}$ in a high-speed centrifuge at $4^{\circ} \mathrm{C}$, and the supernatant was collected to obtain total protein. The protein concentration was measured using a BCA protein assay kit (Beijing Solarbio Science \& Technology Co., Ltd.). The samples were then separated on 10\% SDS-PAGE gels and electro-transferred to polyvinylidene fluoride (PVDF) membranes. The membranes were blocked in Tris-buffered saline (TBS) with 5\% non-fat milk for $2 \mathrm{~h}$ at room temperature, then incubated with primary antibodies [tropomyosin receptor kinase B (TrkB), 1:800, GB11295-1, Wuhan Servicebio Technology Co., Ltd.; GFAP, 1:1,000, GB12096, Wuhan Servicebio Technology Co., Ltd.; NeuN, 1:800, GB11138, Wuhan Servicebio Technology Co., Ltd.; p-PI3K, 1:1,000, 17366, Cell Signaling Technology, Inc.; PI3K, 1:800, 4257, Cell Signaling Technology, Inc.; Akt, 1:800, 4685, Cell Signaling Technology, Inc.; p-Akt, 1:1,000, 4058, Cell Signaling Technology, Inc.] at $4^{\circ} \mathrm{C}$ overnight. Subsequently, the membranes were washed with Tris-buffered saline/Tween-20 (TBST) and incubated with secondary antibodies, then washed with TBS. The secondary antibody used was IRDye $800 \mathrm{CW}$ goat anti-rabbit antibody $(1: 20,000$; LI-COR Biosciences). Finally, labeled proteins were detected using a near-infrared imaging system (LI-COR Biosciences) and analyzed using an image analyzer (Image Studio ${ }^{\mathrm{TM}}$ 
Table II. Basic information on ingredients of Chuanxinlian.

\begin{tabular}{|c|c|c|c|}
\hline Ingredient code & Ingredient name & $\mathrm{OB}(\%)$ & DL \\
\hline MOL000173 & Wogonin & 30.68 & 0.23 \\
\hline MOL002928 & Oroxylin A & 41.37 & 0.23 \\
\hline MOL002932 & Panicolin & 76.26 & 0.29 \\
\hline MOL008203 & Andrographolide & 57.06 & 0.34 \\
\hline MOL008206 & Moslosooflavone & 44.09 & 0.25 \\
\hline MOL008218 & 1-Monoolein & 34.13 & 0.3 \\
\hline MOL008219 & $\begin{array}{l}\text { 3-[2-[(1R,4aS,5R,8aS)-5,8a-dimethyl-2-methylene-5- } \\
\text { methylol-decalin-1-yl]ethyl]-5H-furan-2-one }\end{array}$ & 51.78 & 0.28 \\
\hline MOL008228 & Andrographin & 37.57 & 0.33 \\
\hline MOL008229 & Andrographin F & 33.34 & 0.85 \\
\hline MOL008230 & Andrographidine F_qt & 77.13 & 0.45 \\
\hline MOL008232 & $\begin{array}{l}(3 \mathrm{Z}, 4 \mathrm{~S})-3-[2-[(1 \mathrm{R}, 4 \mathrm{aS}, 5 \mathrm{R}, 6 \mathrm{R}, 8 \mathrm{aS})-6 \text {-hydroxy-5,8a- } \\
\text { dimethyl-2-methylene-5-methylol-decalin-1-yl]ethylidene]-4- } \\
\text { hydroxy-tetrahydrofuran-2-one }\end{array}$ & 46.96 & 0.36 \\
\hline MOL008238 & $\begin{array}{l}\text { 3-[2-[(1S,4aR,5S,8aR)-5,8a-dimethyl-2-methylene-5- } \\
\text { methylol-decalin-1-yl]ethyl]-5H-furan-2-one }\end{array}$ & 63.54 & 0.28 \\
\hline MOL008239 & Quercetin tetramethyl $\left(3^{\prime}, 4^{\prime}, 5,7\right)$ ether & 31.57 & 0.41 \\
\hline
\end{tabular}

OB, oral bioavailability; DL, drug-likeness.

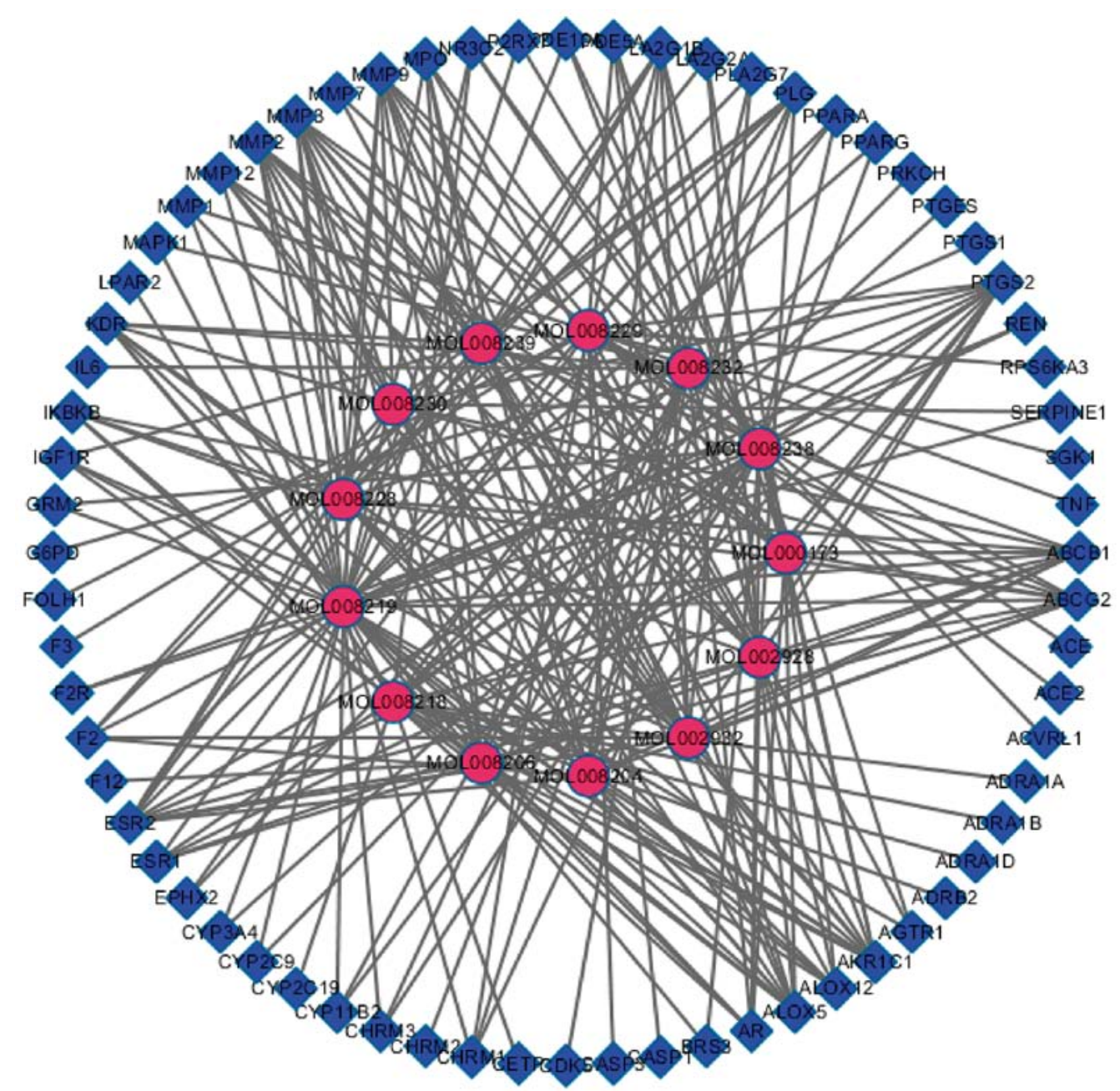

Figure 2. Andrographis paniculata active ingredient/anti-ischemic target network map. The rose-red-colored circles represent the active ingredients of Andrographis paniculata, while the blue-colored squares represent the targets of these active ingredients against cerebral ischemia. 


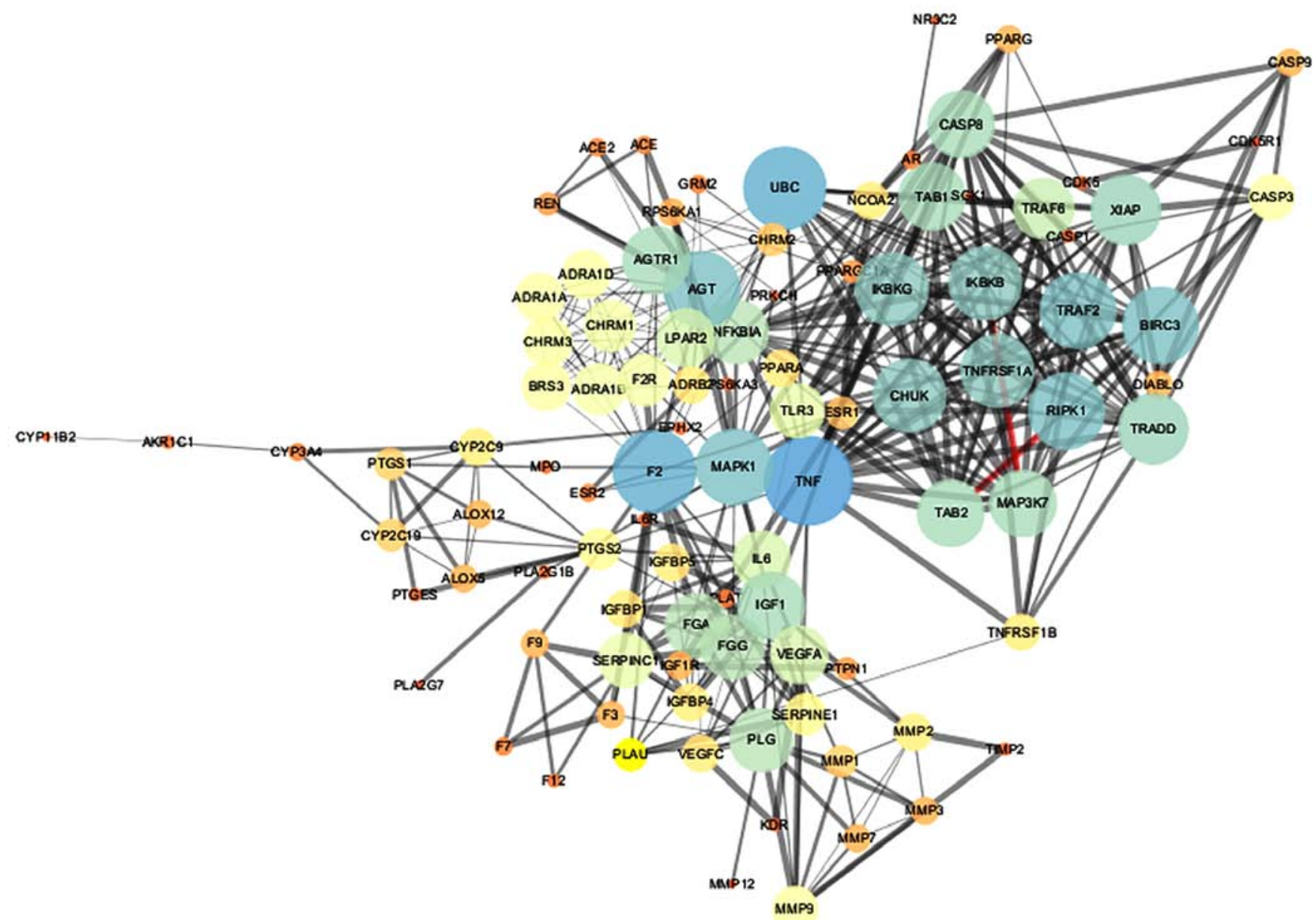

Figure 3. Interaction network of potential targets of Andrographis paniculata for the treatment of ischemic stroke. The 'node' represents the target protein, and the larger the degree value, the larger the node, and the larger the degree value corresponding to the color ranged from blue to red. The 'edge' represents the correlation between the targets, and the thickness of the edge is based on the combined score.

Software). The densitometric values were normalized to the corresponding GAPDH densitometry values.

$R T$ - $q P C R$. Total RNA was extracted from the brain tissues using the TRIzol reagent (R0016, Beyotime Institute of Biotechnology). RNA concentration of the samples was determined by Natodrop 2000 (Thermo Fisher Scientific, Inc.). The total RNA reverse transcription was performed using the PrimeScript ${ }^{\circledR}$ RT Master Mix Perfect Real-Time kit (RR036A, Takara Bio, Inc.). In the following, cDNA was taken as the template for real-time PCR with TB Green ${ }^{\circledR}$ Premix Ex Taq (RR420A, Takara Bio, Inc.). The steps for qPCR included: Initial denaturation by incubating in $95^{\circ} \mathrm{C}$ for $30 \mathrm{sec}$; $40 \mathrm{qPCR}$ cycles by $95^{\circ} \mathrm{C}$ for $5 \mathrm{sec}$ and then $60^{\circ} \mathrm{C}$ for $30 \mathrm{sec}$ in each cycle. Each sample was determined triply and relative expression levels were analyzed using the $2^{-\Delta \Delta \mathrm{Cq}}$ method (17). $\beta$-actin was used for normalization. The sequences of the primers are presented in Table I.

Network pharmacology analyses. Network pharmacology analyses included the following:

i) Establishment of the drug database. Traditional Chinese Medicine Systems Pharmacology (TCMSP) was used to screen, predict and collect the active ingredients of Chuanxinlian using oral bioavailability (OB) and drug-likeness (DL) as the limiting conditions. ii) Targets of CXL active ingredients. The simplified molecular-input line-entry system (SMILES) chemical formulas of all active ingredients of Chuanxinlian obtained in the current screen were downloaded from the PubChem database and the SMILES chemical formulas corresponding to the active ingredients were then entered into the similarity ensemble approach (SEA; http://sea.bkslab.org/) database to obtain therapeutic targets. Finally, the UniProtKB search function in the protein database (http://www.uniprot.org/uniprot/) was applied, and targets and UniProt numbers related to active ingredients were obtained after all retrieved targets were associated to their official symbol by entering the targets name and qualifying the species as human.

iii) Potential disease targets. Multiple databases (TTD, Drugbank and DisGeNET) were searched to collect therapeutic targets related to ischemic stroke using the terms 'Cerebral ischemic stroke', 'Cerebral infarction', 'Ischemic stroke' and 'Acute ischemic stroke'. The database of targets related to ischemic stroke was constructed by comparing and de-weighting the 3 databases.

iv) Protein interaction. The targets of the active ingredients of Chuanxinlian were intersected with the targets implicated in ischemic stroke to obtain the targets of Chuanxinlian potentially involved in treating ischemic stroke, referred to as Chuanxinlian-target-cerebral ischemia. The String database 
Table III. Information on potential targets and topological properties of Chuanxinlian against cerebral ischemia.

\begin{tabular}{rlllrl}
\hline No. & UniProt ID & Gene name & \multicolumn{1}{c}{ Protein name } & Degree & Betweenness \\
\hline 1 & O14920 & IKBKB & $\begin{array}{l}\text { Inhibitor of nuclear factor kappa-B kinase subunit } \\
\text { beta }\end{array}$ & 18 & 0.00467253 \\
2 & P00734 & F2 & Coagulation factor II, thrombin & 11 & 0.00838562 \\
3 & P00747 & PLG & Plasminogen & 15 & 0.05091082 \\
4 & P01375 & TNF & Tumor necrosis factor & 23 & 0.19812513 \\
5 & P03372 & ESR1 & Estrogen receptor 1 & 6 & 0.01264776 \\
6 & P05121 & SERPINE1 & Serpin family E member 1 & 8 & 0.00336983 \\
7 & P07550 & ADRB2 & Adrenoceptor beta 2 & 7 & 0.01043808 \\
8 & P08069 & IGF1R & Insulin-like growth factor 1 receptor & 5 & 0.00441784 \\
9 & P08172 & CHRM2 & Muscarinic acetylcholine receptor M2 & 6 & 0.00606293 \\
10 & P08253 & MMP2 & Matrix metallopeptidase 2 & 7 & 0.01000518 \\
11 & P11712 & CYP2C9 & Cytochrome P450 family 2 subfamily C member 9 & 7 & 0.04508112 \\
12 & P13726 & F3 & Coagulation factor III, tissue factor & 5 & 0.00583017 \\
13 & P14780 & MMP9 & Matrix metallopeptidase 9 & 9 & 0.04962157 \\
14 & P25116 & F2R & Coagulation factor II thrombin receptor & 11 & 0.00838562 \\
15 & P28482 & MAPK1 & Mitogen-activated protein kinase 1 & 19 & 0.21744713 \\
16 & P30556 & AGTR1 & Angiotensin II receptor type 1 & 6 & 0.0567444 \\
17 & P33261 & CYP2C19 & Cytochrome P450 family 2 subfamily C member 19 & 8 & 0.0237056 \\
18 & P35354 & PTGS2 & Prostaglandin-endoperoxide synthase 2 & 5 & 0.04858176 \\
19 & P37231 & PPARG & Peroxisome proliferator-activated receptor gamma & 5 & 0.00262056 \\
20 & P42574 & CASP3 & Caspase-3 & 7 & 0.02624386 \\
21 & Q07869 & PPARA & Peroxisome proliferator-activated receptor alpha & 13 & 0.01747176 \\
22 & Q9HBW0 & LPAR2 & Lysophosphatidic acid receptor 2 & & \\
\hline
\end{tabular}

(https://string-db.org/) (17), a database containing known and predicted protein-protein interactions, was used.

The protein targets of Chuanxinlian were imported into the String database to obtain their interaction associations, qualifying the species as human. The results were saved in TSV format, and the nodes (node 1, node 2) and combined score information in the file were reserved, then imported into Cytoscape 3.2.1 to draw the interaction network, and the network results were analyzed and saved. Using the 'generate style from statistics' tool in Cytoscape, the node size and color were set to reflect the number of components connected to the target (degree), and the thickness of edge setting was set to reflect the size of the combined core. Finally, the ultimate protein interaction network was obtained.

v) Construction of active ingredient-target network diagram. The active ingredients and targets of Cuanxinlian were imported into Cytoscape 3.2.1 software and the ingredient-target network diagram of Chuanxinlian was constructed to predict the main targets and active ingredients of Chuanxinlian involved in treating cerebral ischemia.

vi) Analysis of biological processes and pathways. The targets of Chuanxinlian were input into the DAVID database (18), with the selected Identifier set to the OFFICIAL GENE SYMBOL, List Type set to the GENE List, and species limited to human. Gene ontology (GO) functional enrichment analysis and Kyoto Encyclopedia of Genes and Genomes (KEGG) pathway enrichment analysis were carried out for the targets of Andro. Its mechanism was investigated and the results were saved. The top biological processes and signaling pathways were obtained by setting $\mathrm{P}<0.05$.

Statistical analysis. All data are expressed as the means \pm standard deviation and analyzed using SPSS 17.0 (SPSS, Inc.). One-way analysis of variance and the Bonferroni post hoc test were used to evaluate statistical significance. Results were considered statistically significant when $\mathrm{P}<0.05$.

\section{Results}

Components of Chuanxinlian and their corresponding target proteins. Through the TCMSP database and literature investigation, 49 chemical components of Chuanxinlian were obtained. Using $\mathrm{OB} \geq 30 \%$ and $\mathrm{DL} \geq 0.18$ as the filter criteria, 13 active ingredients were found to meet the requirements. Basic information on the ingredients is presented in Table II.

Target prediction. All targets were obtained from the TCMSP, SEA and SIA databases, and the duplicates and false positives were removed. Subsequently, 428 targets of Chuanxinlian were integrated; 421 ischemic stroke symptom targets were obtained from the DisGeNET, Drugbank and TTD databases; in addition, 68 potential targets were obtained from the intersection of Chuanxinlian targets and ischemic stroke targets (Table II).

Active ingredient-target network. Cytoscape 3.2.1 was used to construct the Andrographis paniculata active 
A

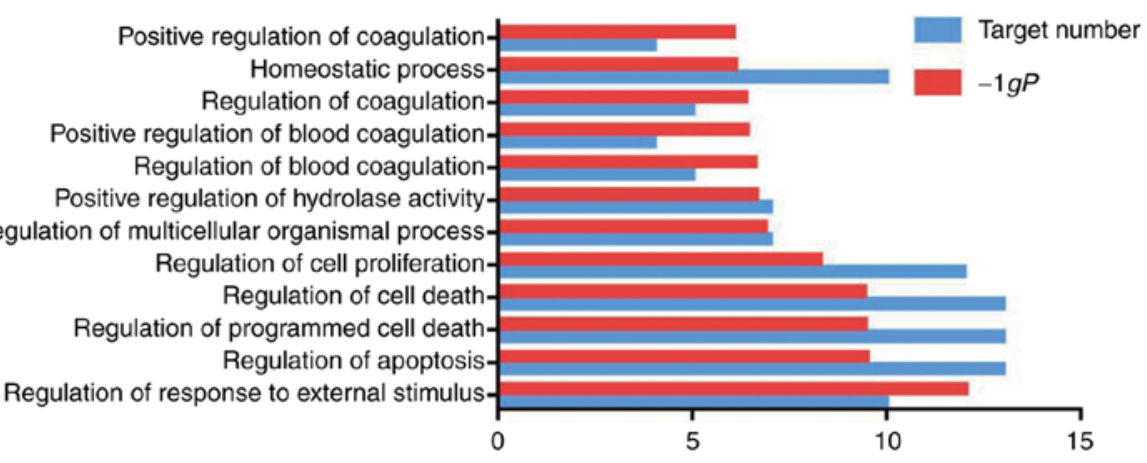

B

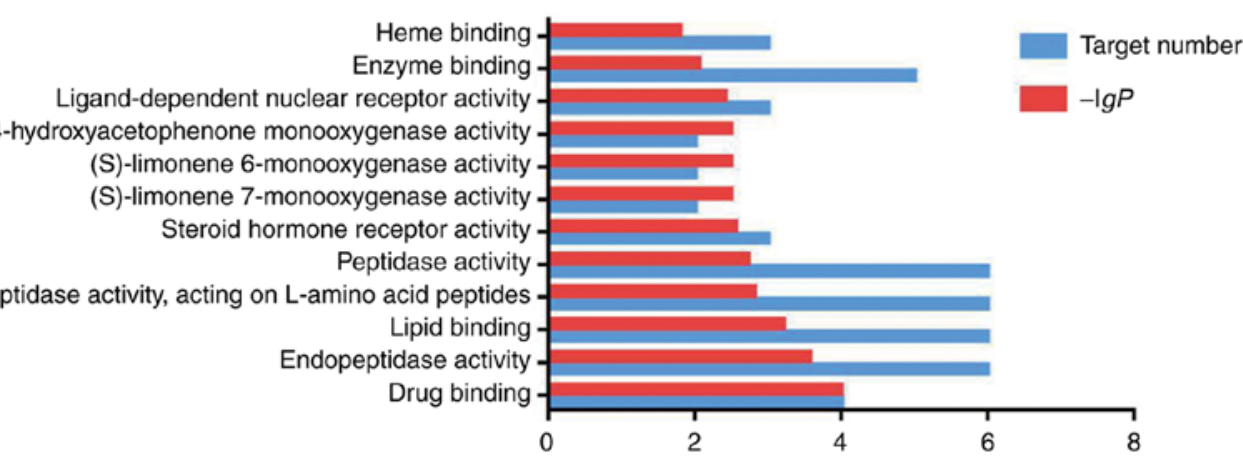

C
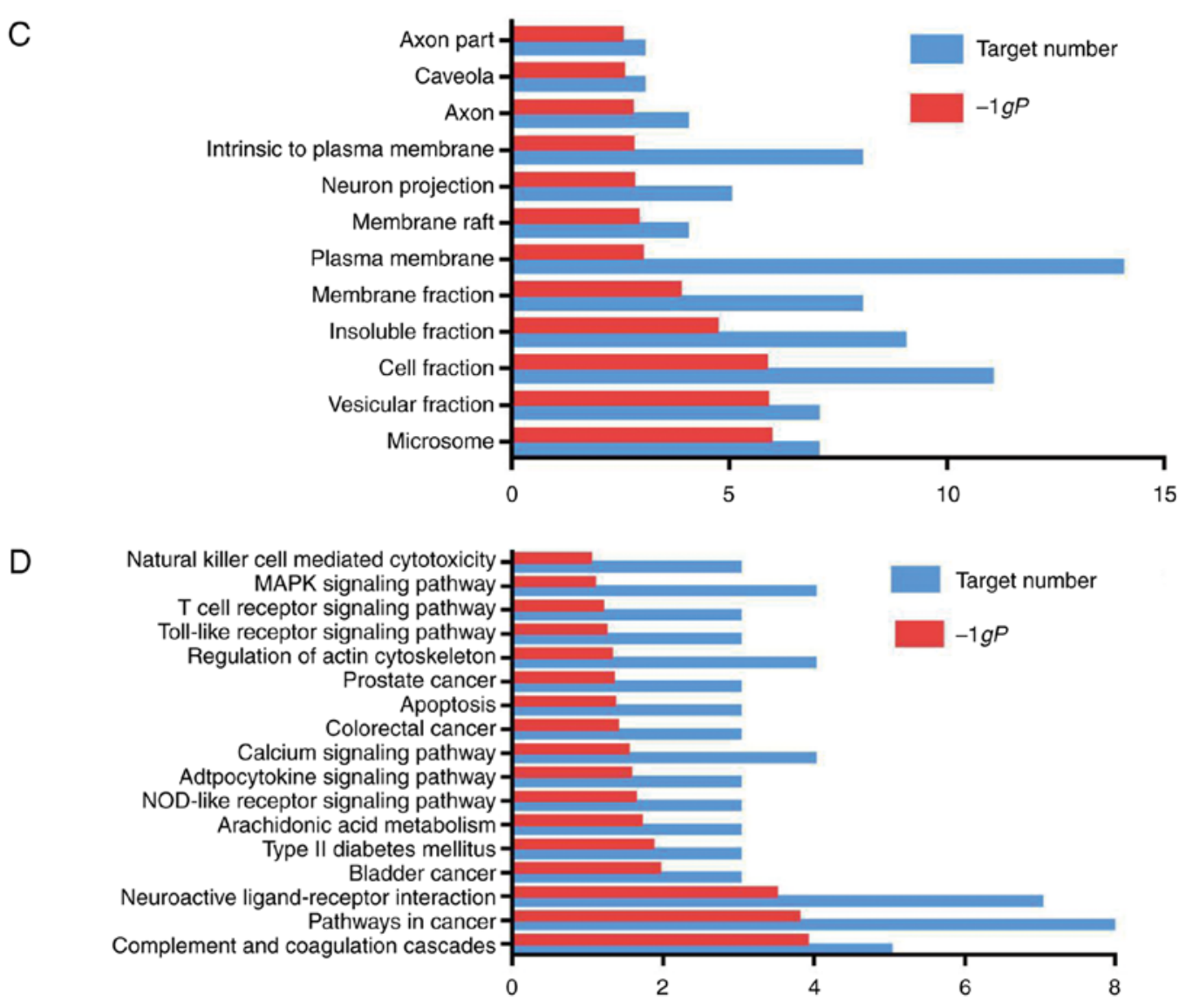

Figure 4. Interaction network of potential targets of Chuanxinlian for the treatment of ischemic stroke. (A) Enriched gene ontology terms for biological processes, (B) molecular functions, (C) cell components, and (D) KEGG pathway enrichment analysis of potential targets of the main active ingredients of Chuanxinlian.

ingredient-anti-ischemic target map (Fig. 2), which contained 81 nodes and 249 edges. The rose-red-colored circles represent the active ingredients of Chuanxinlian, while the blue-colored squares represent the targets of these active ingredients against cerebral ischemia, and the edge represents the interaction between the active ingredient and anti-cerebral ischemia targets. 


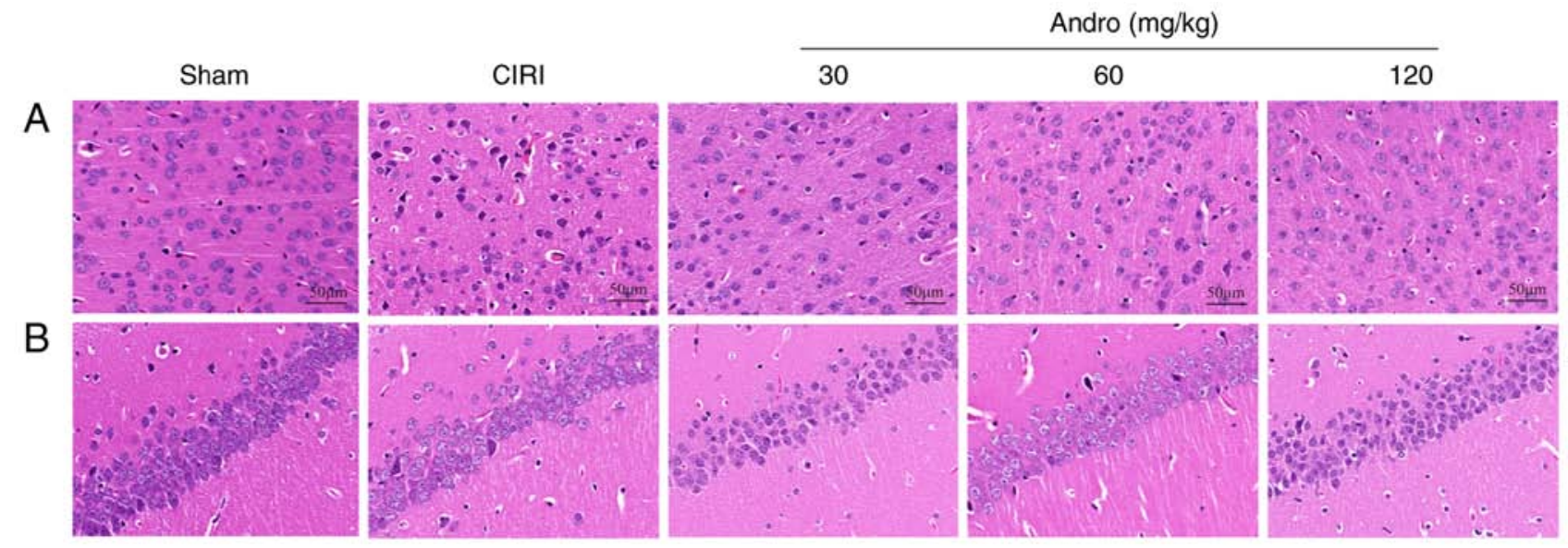

Figure 5. Effect of andrographolide pre-treatment on pathological changes of the (A) brain cortex and (B) hippocampal CA1 region following CIRI (H\&E staining; magnification, $\mathrm{x} 200$; scale bar, $50 \mu \mathrm{m} ; \mathrm{n}=3$ ). Pre-treatment with 30,60 and $120 \mathrm{mg} / \mathrm{kg}$ andrographolide reduced the histopathological alteration following CIRI to a certain degree. Sham, sham-operated group; CIRI, cerebral ischemia-reperfusion injury; Andro, andrographolide.

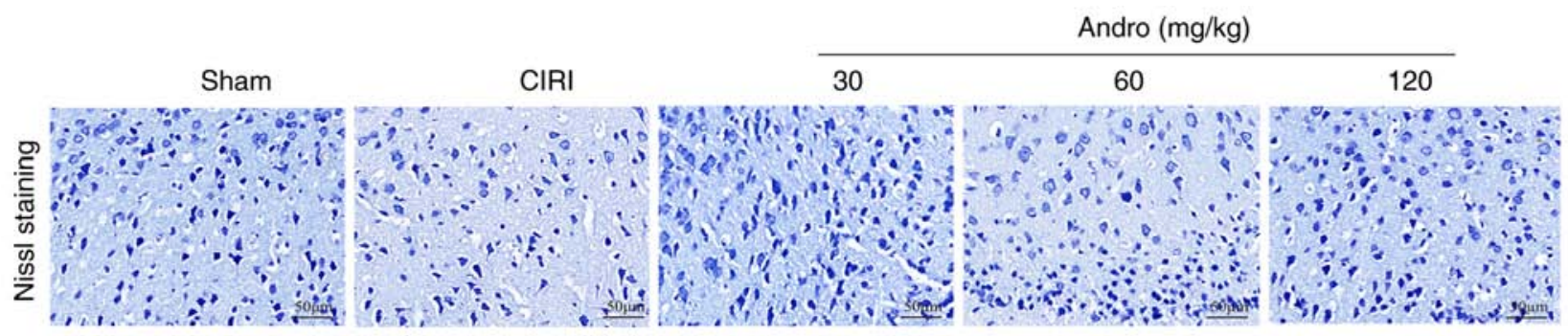

Figure 6. Effect of andrographolide pre-treatment on changes in Nissl staining in the brain cortex following CIRI (Nissl staining; magnification, x200; scale bar, $50 \mu \mathrm{m}$; $\mathrm{n}=3$ ). Sham, sham-operated group; CIRI, cerebral ischemia-reperfusion injury; Andro, andrographolide.

Protein interaction network diagram. Target interactions were obtained from the String database, using Cytoscape 3.2.1 to draw the network diagram (Fig. 3). The 'edge' represents the correlation between the targets, and the thickness of the edge corresponds to the combined score: The thicker the edge, the greater the value of the combined score and the greater the degree of association. The node represents the target, and the value of 'degree' represents the effect intensity. The larger the degree value, the larger the node, and the larger the degree value corresponding to the color ranged from blue to red. There were 98 nodes and 1,099 edges in the figure. The average node degree was 20.4 and the average local clustering coefficient was 0.624 . Finally, nodes with degree $\geq 5$ and betweenness $\geq 0.00262056$ were selected as the main nodes; thus, there were a total of 22 main nodes, as listed in Table III.

Gene function and pathway analysis. Gene function and pathway analysis revealed the following results:

i) GO enrichment analysis. The GO enrichment function and the KEGG pathway in DAVID were used to analyze the 22 selected targets. GO enrichment analysis includes 3 branches: Biological processes, molecular function and cellular component. At the $\mathrm{P}<0.05$ setting, 298 biological processes or pathways were obtained. Biological processes or pathways with a higher P-value were screened, and GraphPad Prism7.0 was used for mapping.

As shown in Fig. 4A, the main biological processes of the targets were the regulation of response to an external stimulus, regulation of apoptosis and the regulation of programmed cell death. As shown in Fig. 4B, the main molecular functions of the targets were drug binding, endopeptidase activity, lipid binding and peptidase activity. Plasminogen (PLG) is a single-chain glycoprotein that plays an important role in thrombolytic therapy for acute cardiogenic cerebral infarction. When stimulated, vascular endothelial cells secrete a large amount of tissue-type plasminogen activator, which converts PLG into fibrinase and this activates the fibrinolytic system in vivo. Some autosomal genes associated with ischemic heart disease, including the gene encoding PLG, have been identified (19). PLG is also a key molecule involved in the thrombolytic treatment of cerebral ischemia. As shown in Fig. 4C, the cell components of the targets included cell fraction, plasma membrane and neuron projection.

ii) Pathway analysis. The results of KEGG pathway enrichment analysis are shown in Fig. 4D. The targets identified were associated with the NOD-like receptor, mitogen-activated protein kinase (MAPK) and phosphoinositide 3-kinase (PI3K)/Akt and Toll-like receptor signaling pathways. The results revealed that the main active ingredients of Chuanxinlian were largely distributed into different metabolic pathways. Thus, the mechanisms through which Chuanxinlian protects against damage following ischemic stroke may involve multiple ingredients, multiple targets and multiple pathways.

Andro attenuates the pathological abnormalities caused by CIRI as shown by H\&E staining. The brain sections of the 
A
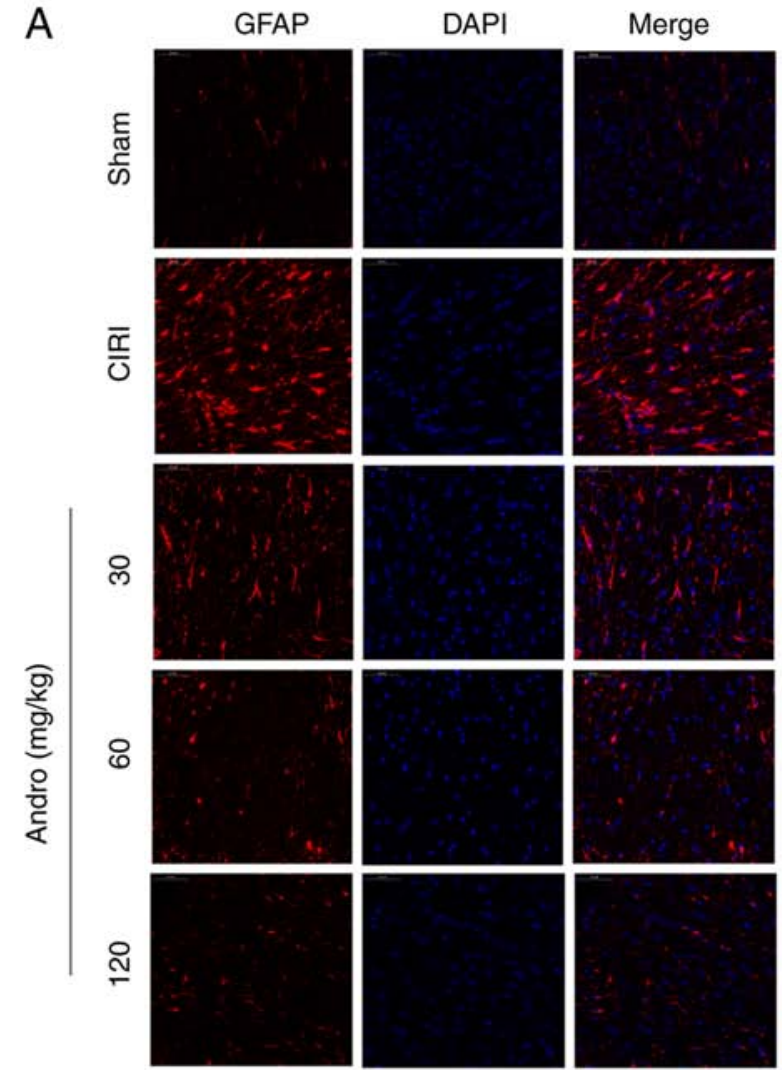

C

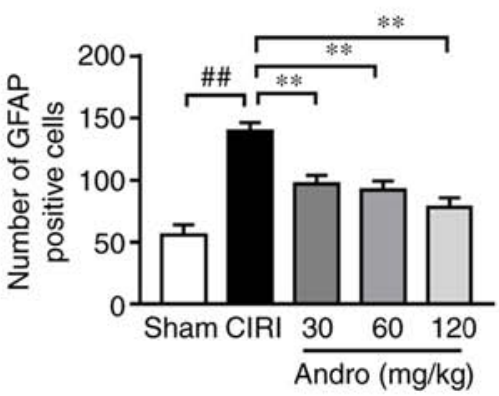

B
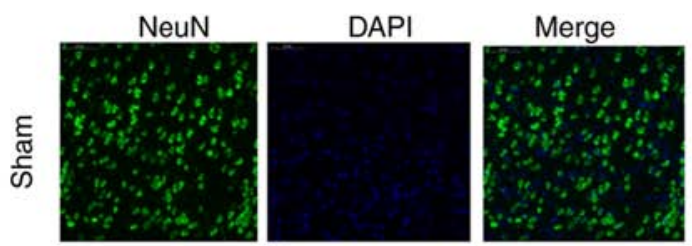

요
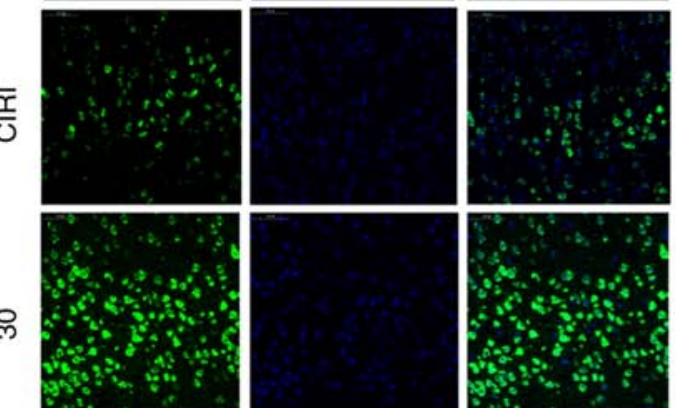

8
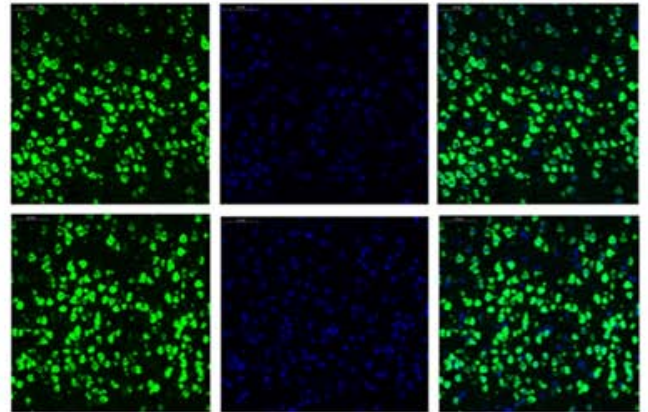

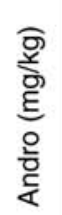

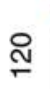
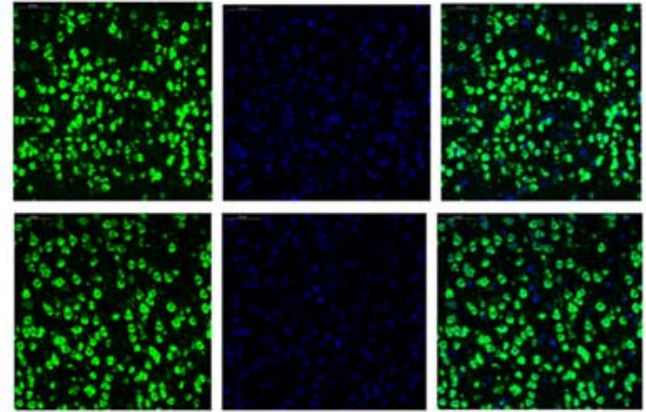

D

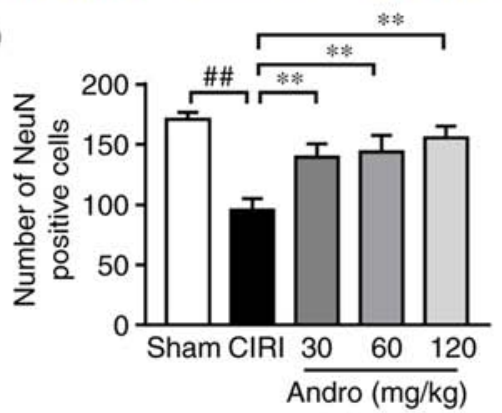

Figure 7. Influence of Andro on protein expression levels of (A) GFAP and (B) NeuN in brains of mice subjected to CIRI based on immunofluorescence staining. DAPI was used to stain the nuclei blue, GFAP-positive cells are indicated in red, and NeuN-positive cells are indicated in green. (C) Quantification of GFAP-positive cells. (D) Quantification of NeuN-positive cells. Data are presented as the means \pm standard deviation (n=3). ${ }^{\# \#} \mathrm{P}<0.01$ vs. sham group; ${ }^{* *} \mathrm{P}<0.01$ vs. CIRI group. Scale bar, $50 \mu \mathrm{m}$. GFAP, glial fibrillary acidic protein; NeuN, neuronal nuclei; sham, sham-operated group; CIRI, cerebral ischemia-reperfusion injury; Andro, andrographolide.

mice were stained with H\&E (Fig. 5), and the results revealed that neurons in the cerebral cortex and hippocampal CA1 region in the sham group were normal. In these mice, brain tissue was not damaged and no obvious vacuolar space was observed. The nerve cells were densely and evenly arranged, with normal structure and morphology, clear cell contour, complete normal morphology and clearly visible nucleoli. By contrast, in the CIRI group, ischemic necrosis occurred in the cerebral cortex, with the shrinkage of neuronal nuclei; some cells exhibited spot-like necrosis, with evident infiltration of microglial cells, neuronal disorder and tissue edema in the hippocampus.

Andro increases Nissl positive staining. The mouse brain sections were stained with Nissl (Fig. 6), and the results revealed that, compared with the sham group, the numbers of Nissl-positive cells in the CIRI group were markedly decreased. Following pre-treatment with Andro, the numbers of Nissl-positive cells were significantly increased compared to the CIRI group.

Andro decreases GFAP and increases NeuN expression, as shown by immunofluorescence staining. The results of immunofluorescence staining illustrated that, compared with the sham group, the expression of GFAP was significantly increased (Fig. 7A and C) and the expression of NeuN was significantly decreased (Fig. 7B and D) in the brains of the mice subjected to CIRI. Following pre-treatment with Andro, the expression of GFAP significantly decreased, while the expression of NeuN significantly increased.

Andro increases the levels of BDNF. BDNF is involved in the development of the nervous system, and CIRI promotes the release of BDNF. Compared with the sham group, the BDNF 
A

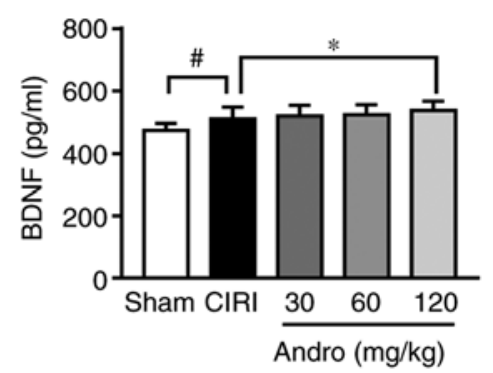

D
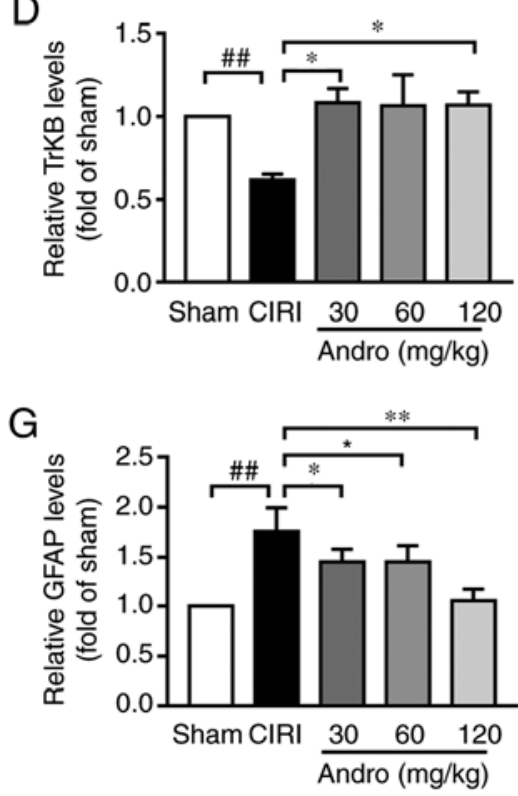

B

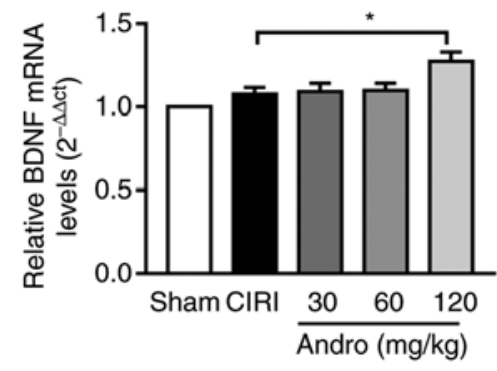

$\mathrm{E}$

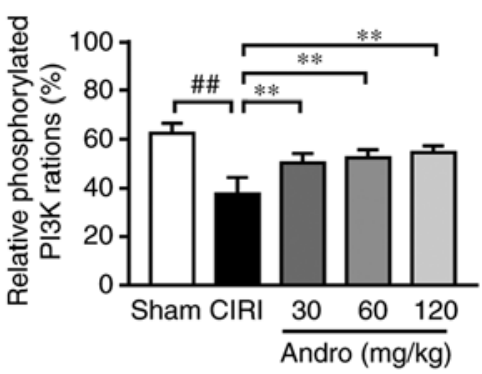

$\mathrm{H}$

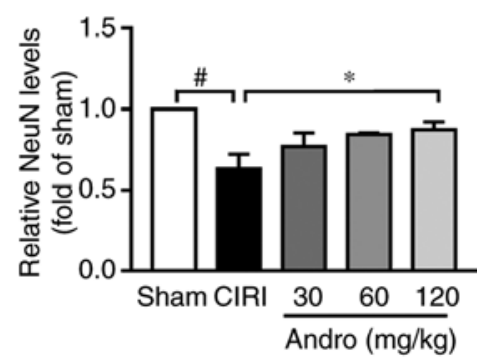

C
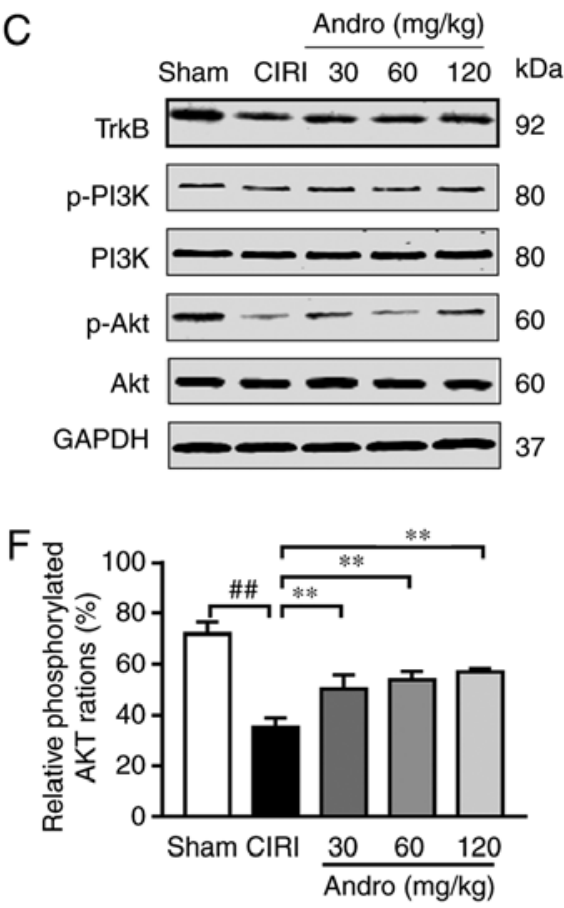

I

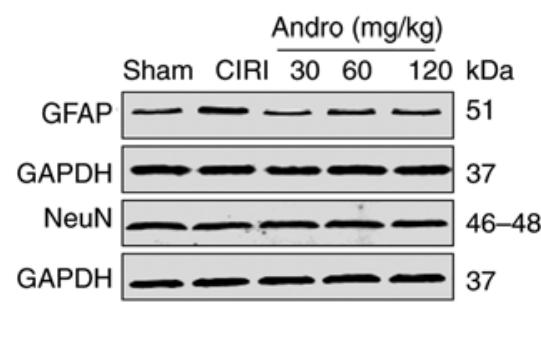

Figure 8. Influence of Andro on BDNF, TrkB, GFAP, NeuN, p-PI3K and p-Akt in mouse brains following CIRI was examined. Mice were treated with Andro (30,60, or $120 \mathrm{mg} / \mathrm{kg}$ ) for 14 days prior to CIRI, and repeated CIRI was then performed. BDNF was detected by (A) enzyme-linked immunosorbent assay and (B) RT-qPCR. (C-I) Protein levels of TrkB, p-PI3K, PI3K, p-Akt, Akt, GFAP and NeuN in brains of CIRI mice were assessed by western blot analysis. ${ }^{\#} \mathrm{P}<0.05,{ }^{\# \#} \mathrm{P}<0.01$ vs. sham group; ${ }^{*} \mathrm{P}<0.05,{ }^{* *} \mathrm{P}<0.01$ vs. CIRI group BDNF, brain-derived neurotrophic factor; TrkB, tropomyosin receptor kinase $\mathrm{B}$; GFAP, glial fibrillary acidic protein; NeuN, neuronal nuclei; p-PI3K, phosphorylated phosphoinositide 3-kinase; Sham, sham-operated group; CIRI, cerebral ischemia-reperfusion injury; Andro, andrographolide.

content in the serum of mice in the CIRI group was significantly increased $(\mathrm{P}<0.05)$, and the $B D N F$ mRNA level in the brain tissues also exhibited an increasing tendency (Fig. 8A and B). This indicates that cerebral ischemia-reperfusion can stimulate the release of BDNF in a short period of time. Compared with the CIRI group, the serum BDNF levels were significantly increased in mice pre-treated with Andro $(\mathrm{P}<0.05)$, and the $B D N F$ mRNA levels in the brain tissues of mice receiving CIRI also exhibited an increasing trend. In addition, Andro pre-treatment enhanced the transcription of BDNF in mice subjected to CIRI.

Andro increases the levels of TrkB, and downstream proteins. TrkB is a primary receptor of BDNF. Compared with the sham group, TrkB protein expression in the brain tissues of mice in the CIRI group was significantly decreased $(\mathrm{P}<0.01)$, and compared with the CIRI group, the mice that received Andro $(120 \mathrm{mg} / \mathrm{kg})$ pre-administration exhibited a significantly upregulated TrkB protein expression in the brain tissue $(\mathrm{P}<0.01$; Fig. $8 \mathrm{C}$ and $\mathrm{D})$.
The levels of proteins downstream of TrkB [including phosphorylated PI3K (p-PI3K) and phosphorylated Akt (p-Akt) were reduced in the mice in the CIRI group, while Andro pre-treatment markedly increased the phosphorylation levels of these proteins compared to the CIRI group (Fig. 8C, E and F).

GFAP is a specific marker of activated astrocytes, and NeuN is a specific marker of mature neurons. CIRI increased the expression of GFAP and inhibited the expression of NeuN, while Andro administration markedly reduced the expression of GFAP and enhanced the expression of NeuN (Fig. 8G-I).

Andro reduces the levels of pro-inflammatory cytokines. ELISA was used to determine the effects of Andro on the levels of the inflammatory cytokines, $I L-1 \beta, I L-6$ and $T N F-\alpha$ in serum. The serum levels of $I L-1 \beta$ and $T N F-\alpha$ were significantly increased in the CIRI group compared to the sham group. However, compared with the CIRI group, the serum levels of $I L-1 \beta, I L-6$ and $T N F$ - $\alpha$ were markedly decreased in the Andro groups (Fig. 9A-C). 
A
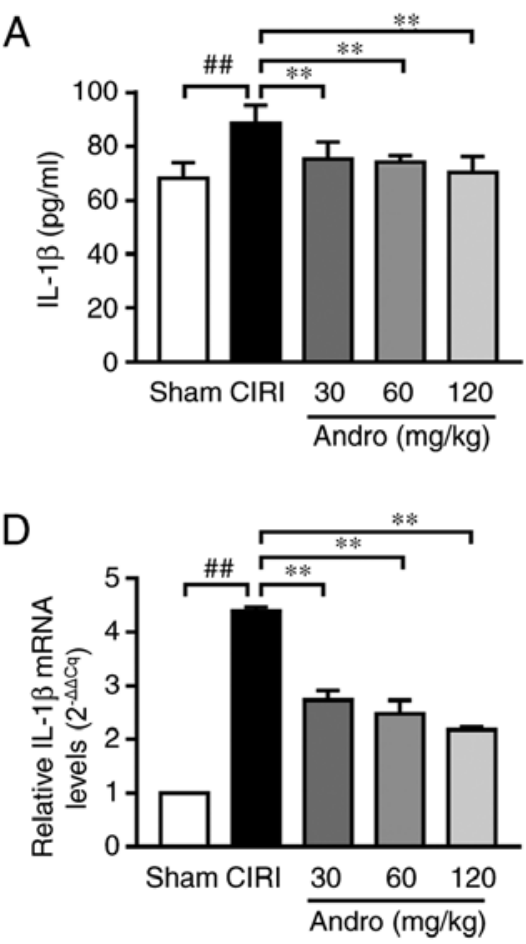

B

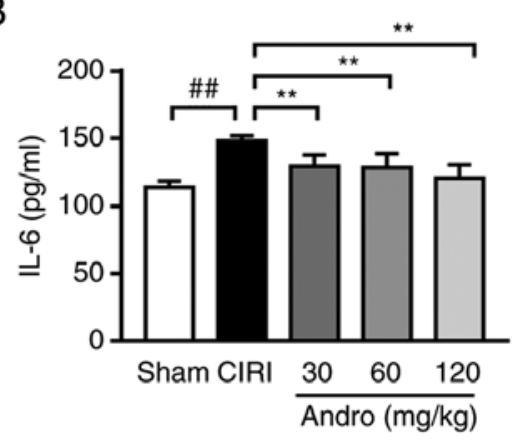

E

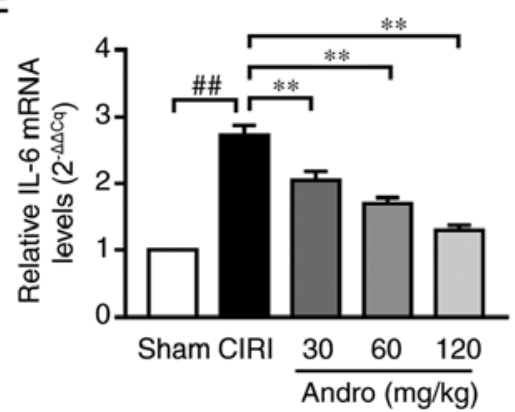

C

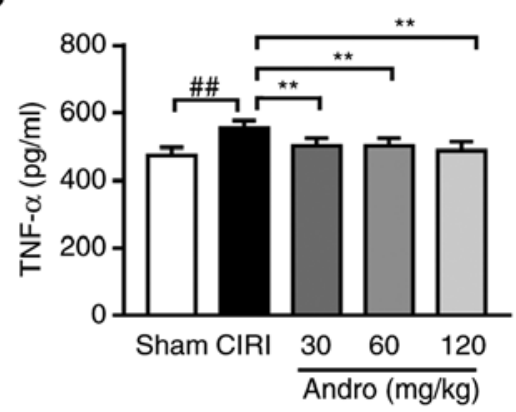

F

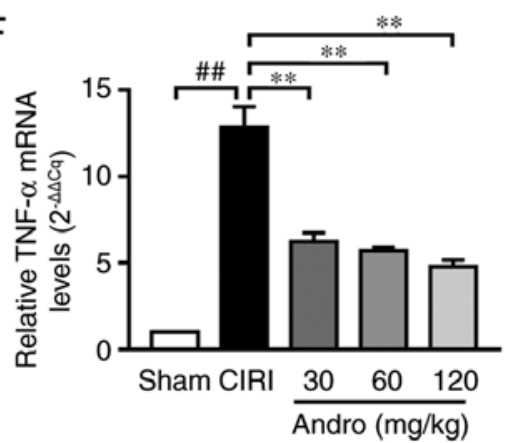

Figure 9. Influence of Andro on inflammatory cytokine levels in mouse serum and brain following CIRI was examined. Enzyme-linked immunosorbent assay was used to examine serum levels of inflammatory cytokines (A) IL-1 $\beta$, (B) IL-6, and (C) TNF- $\alpha$ in mice subjected to CIRI with or without Andro pre-treatment. (D-F) RT-q-PCR was used to measure the mRNA expression levels of (D) IL-1 $\beta$, (E) IL-6, and (F) TNF- $\alpha$ in the brain tissues of mice subjected to CIRI. Data are presented as the means \pm standard deviation $(n=3) .{ }^{\# \#} \mathrm{P}<0.01$ vs. sham group; ${ }^{* *} \mathrm{P}<0.01$ vs. CIRI group. IL, interleukin; TNF- $\alpha$, tumor necrosis factor $\alpha$; Sham, sham-operated group; CIRI, cerebral ischemia-reperfusion injury; Andro, andrographolide.

In addition, the $I L-1 \beta$ mRNA, $I L-6$ mRNA and $T N F-\alpha$ mRNA levels in brain samples were analyzed by RT-qPCR (Fig. 9D-F). Compared with the sham group, the $I L-1 \beta$ mRNA, $I L-6$ mRNA and $T N F-\alpha$ mRNA levels were significantly increased in the CIRI group. In addition, pre-treatment with Andro significantly downregulated the $I L-1 \beta$ mRNA, $I L-6$ mRNA and TNF- $\alpha$ mRNA levels (Fig. 9D-F).

\section{Discussion}

Stroke is the third-leading cause of mortality in several developed countries and is associated with high morbidity, disability and mortality (20). Inflammation and apoptosis, as the critical factors involved in CIRI, can interact with each other and further create a destructive cascade effect (21). When brain tissue is damaged, the levels of pro-inflammatory cytokines (IL-1 $\beta, I L-6$ and $T N F-\alpha$ ) increase. Due to the high incidence and severe consequences of ischemic stroke, new drugs are urgently required for the treatment of ischemic stroke (22).

The present study mainly aimed to predict and analyze the target and pathway of Andrographis paniculata through network pharmacology. From previous studies $(6,11,12)$, Andro has been demonstrated to possess potent neuroprotective action in the treatment of neurological injury and cognitive dysfunction. Andro is one of the main components of Andrographis paniculata. Therefore, Andro was used to further verify the results, and other components could also be selected for subsequent experiments.

Andrographis paniculata, an agent used in TCM, has the effect of clearing heat and removing toxicity, and clearing heat and removing toxicity is one of the 8 treatment methods of TCM. Andro is a terpene that has been used extensively in China for the treatment of a range of diseases. Previous studies have illustrated that Andro significantly inhibits free radical formation, blood-brain barrier destruction and cerebral infarction in rats subjected to MCAO, and the heme oxygenase 1 (HO-1) inhibitor zinc protoporphyrin IX reverses these effects, indicating that Andro increases Nrf2-HO-1 expression through the regulation of p38 MAPK, confirming that it protects against MCAO-induced brain injury (23). Moreover, Andro reduces the expression of NADPH oxidase 2 (NOX2) and inducible nitric oxide synthase (iNOS), possibly by impairing PI3K/Akt-dependent NF- $\mathrm{BB}$ and hypoxia-inducible factor (HIF)- $1 \alpha$ activation. This affects microglial activation, which in turn mediates the protective effects of Andro in mice subjected to CIRI (24).

The present study aimed to explore the neuroprotective effects of Andro on CIRI. The data demonstrated that the administration of Andro attenuated pathological changes and increased the number of Nissl-positive cells. Moreover, immunofluorescence staining confirmed that Andro effectively reduced the expression of GFAP and increased the expression of NeuN in mice following CIRI. Nissl bodies, which exist in the cytoplasm of neurons, are characteristic structures of neurons, and their role is to synthesize structural proteins, enzymes, and nerve modulators for neurons. When neurons undergo pathological changes, Nissl bodies begin to dissolve and become dust-like particles that spread out from the nucleus until they disappear (25). NeuN is a specific marker of mature neuronsand is expressed only after the neuron matures, with 
its expression increasing gradually. Therefore, the expression of NeuN can reflect the maturity of neurons (26). GFAP is a specific marker of activated astrocytes (27). Astrocytes, which are a substantial component of nerve cells, are less sensitive to ischemic hypoxia (28). They are activated when cerebral ischemia and other injuries occur (28).

The results of the present study illustrate that Andro protects the brain from CIRI through a reduction in the levels of apoptosis-related proteins by activating the BDNF-TrkB/PI3K/Akt signaling pathway. It has been demonstrated that BDNF promotes post-CIRI neurological recovery, prevents neuron death and supports the survival of multiple neurons (29). BDNF is mainly synthesized and secreted by presynaptic and postsynaptic neurons and may mediate axonal growth and brain plasticity (30). BDNF activates downstream signaling pathways such as the PI3K/Akt pathway through binding to its cell surface receptor TrkB after being secreted into the extracellular space (31). It has been confirmed that the PI3K/Akt signaling pathway, a significant cell signal transduction pathway, plays an essential role in regulating cell survival, apoptosis, necrosis and other pathophysiological processes (32). The suppression of Akt activity has been associated with neuronal cell death following I/R injury (33), and a previous study demonstrated that Akt was activated by Ser473 phosphorylation (34). The results of the present study demonstrated that following CIRI, the phosphorylation of both PI3K and Akt was increased, indicating that this signal transduction pathway was activated.

The findings of the present study provide novel insight into a potential method of protection against cerebral ischemia, as well as the mechanism through which Andro exerts its neuroprotective effects. Multiple factors and pathways may be responsible for the therapeutic effects of Andro, including inhibiting inflammation and mediating cell apoptosis. Future studies are required to focus on other pathways that are likely involved in this process. It is difficult to identify the true target proteins of Andro and to measure binding affinity. Future studies may focus on verifying the effects reported herein, particularly through the use of knockout mice. Moreover, proteomics and transcriptomics techniques can also be used to find differential targets. The PI3K/Akt pathway is an important neuroprotective and anti-apoptotic pathway. These findings provide novel insight into a potential method of protection against cerebral infarction, as well as the mechanisms through which Andro exerts its neuroprotective effects.

In the present study, the protective effects of Andro were assessed in a mouse model of CIRI, and as shown in the pathological section, the CIRI of neurons was significantly attenuated. Pre-treatment with Andro upregulated the protein expression of TrkB, p-PI3K and p-Akt. It also decreased the expression of GFAP and increased the expression of NeuN. Furthermore, Andro attenuated inflammation significantly by reducing the serum levels of $I L-1 \beta, I L-6$ and $T N F-\alpha$.

In conclusion, the present study demonstrates that Andro can protect brain tissue from ischemia-reperfusion injury in mice by reducing the inflammatory response and apoptosis of brain tissue cells. It was found that Andro exerts this neuroprotective effect by regulating the expression of important proteins in the PI3K/Akt pathway, indicating that this signaling pathway may be the mechanism behind this protective effect.
The results of the present study suggest that Andro may be an effective agent for the treatment of CIRI. Moreover, the findings presented herein provide a potential explanation for the clinical anti-apoptotic and neuroprotective effects of Andro.

\section{Acknowledgements}

Not applicable.

\section{Funding}

The present study was supported by grants from the National Science and Technology Major Project of 'Development Program of Significant New Drug' (no. 2017ZX09301071), the Scientific and Technological Project of Henan Province (no. 17210231004), the Key Scientific Research Projects of Colleges and Universities in Henan Province (no. 21A360021), Natural Science Foundation of Henan Province (no. 202300410259) and the Henan Province Postdoctoral Research Startup Project (no. 202001043).

\section{Availability of data and materials}

All data generated or analyzed during this study are included in this published article or are available from the corresponding author on reasonable request.

\section{Authors' contributions}

YL, LLX, JXM, CW and MSM conceived and designed the all experiments. YL, LLX and JXM performed the experiments. YL, LLX and CW analyzed the data. YL wrote and edited the manuscript. JXM and MSM revised the manuscript. YL and LLX confirm the authenticity of all the raw data. All authors have read and approved the final manuscript.

\section{Ethics approval and consent to participate}

The present study was performed in accordance with the recommendations of the Ministry of Science and Technology of China's Guidance for the Care and Use of Laboratory Animals. All procedures were approved by the Laboratory Animal Ethics Committee of Henan University of Chinese Medicine. (the number of approval ethics code: DWLL201903026).

\section{Patient consent for publication}

Not applicable.

\section{Competing interests}

The authors declare that they have no competing interests.

\section{References}

1. Krishnamurthi RV, Feigin VL, Forouzanfar MH, Mensah GA, Connor M, Bennett DA, Moran AE, Sacco RL, Anderson LM, Truelsen T, et al: Global and regional burden of first-ever ischaemic and haemorrhagic stroke during 1990-2010: Findings from the global burden of disease study 2010. Lancet Glob Health 1: e259-e281, 2013 
2. Miralbell J, López-Cancio E, López-Oloriz J, Arenillas JF, Barrios M, Soriano-Raya JJ, Galán A, Cáceres C, Alzamora M, Pera G, et al: Cognitive patterns in relation to biomarkers of cerebrovascular disease and vascular risk factors. Cerebrovasc Dis 36: 98-105, 2013.

3. Doyle KP, Simon RP and Stenzel-Poore MP: Mechanisms of ischemic brain damage. Neuropharmacology 55: 310-318, 2008.

4. Song W, Zhao J, Yan XS, Fang X, Huo DS, Wang H, Jia JX and Yang ZJ: Mechanisms associated with protective effects of ginkgo biloba leaf extracton in rat cerebral ischemia reperfusion injury. J Toxicol Environ Health A 82: 1045-1051, 2019.

5. Becker KJ and Buckwalter M: Stroke, inflammation and the immune response: Dawn of a new era. Neurotherapeutics 13: 659-660, 2016

6. Liu T, Yang K, Li G, Zhou K, Tan J, Chen J, Li T, Yu Y and Ning W: Experimental evidence and network pharmacology identify the molecular targets of Tong Sheng tablets in cerebral ischemia reperfusion injury. Am J Transl Res 11: 3301-3316, 2019.

7. Iadecola $\mathrm{C}$ and Anrather J: The immunology of stroke: From mechanisms to translation. Nat Med 17: 796-808, 2011

8. Benjamin EJ, Blaha MJ, Chiuve SE, Cushman M, Das SR, Deo R, de Ferranti SD, Floyd J, Fornage M, Gillespie C, et al: Heart disease and stroke statistics-2017 update: A report from the American heart association. Circulation 135: e146-e603, 2017.

9. Powers WJ and Rabinstein AA: Response by powers and rabinstein to letter regarding article, '2018 guidelines for the early management of patients with acute ischemic stroke: A guideline for healthcare professionals from the American heart association/ American stroke association'. Stroke 50: e277-e278, 2019.

10. Muresanu DF, Strilciuc S and Stan A: Current drug treatment of acute ischemic stroke: Challenges and opportunities. CNS Drugs 33: 841-847, 2019.

11. Chan SJ, Wong WS, Wong PT and Bian JS: Neuroprotective effects of andrographolide in a rat model of permanent cerebral ischaemia. Br J Pharmacol 161: 668-679, 2010

12. Wang DP, Yin H, Lin Q, Fang SP, Shen JH, Wu YF, Su SH and Hai J: Andrographolide enhances hippocampal BDNF signaling and suppresses neuronal apoptosis, astroglial activation, neuroinflammation, and spatial memory deficits in a rat model of chronic cerebral hypoperfusion. Naunyn Schmiedebergs Arch Pharmacol 392: 1277-1284, 2019.

13. Yuan H, Ma Q, Cui H, Liu G, Zhao X, Li W and Piao G: How can synergism of traditional medicines benefit from network pharmacology? Molecules 22: 1135, 2017

14. Ning K, Zhao X, Poetsch A, Chen WH and Yang J: Computational molecular networks and network pharmacology. Biomed Res Int 2017: 7573904, 2017.

15. Zhang JJ, Gao TT, Wang Y, Wang JL, Guan W, Wang YJ, Wang CN, Liu JF and Jiang B: Andrographolide exerts significant antidepressant-like effects involving the hippocampal BDNF system in mice. Int J Neuropsychopharmacol 22: 585-600, 2019.

16. Livak KJ and Schmittgen TD: Analysis of relative gene expression data using real-time quantitative PCR and the 2(-Delta Delta C(T)) method. Methods 25: 402-408, 2001.

17. Fan M, Song C, Wang T, Li L, Dong Y, Jin W and Lu P: Protective effects of lithium chloride treatment on repeated cerebral ischemia-reperfusion injury in mice. Neurol Sci 36: 315-321, 2015.

18. Huang DW, Sherman BT, Tan Q, Kir J, Liu D, Bryant D, Guo Y, Stephens R, Baseler MW, Lane HC and Lempicki RA: DAVID bioinformatics resources: Expanded annotation database and novel algorithms to better extract biology from large gene lists. Nucleic Acids Res 35 (Web Server Issue): W169-W175, 2007.

19. Schooling CM, Huang JV, Zhao JV, Kwok MK, Au Yeung SL and Lin SL: Disconnect between genes associated with ischemic heart disease and targets of ischemic heart disease treatments. EBioMedicine 28: 311-315, 2018.
20. van Hoof RHM, Schreuder FHBM, Nelemans P, Truijman MTB, van Orshoven NP, Schreuder TH, Mess WH, Heeneman S, van Oostenbrugge RJ, Wildberger JE and Kooi ME: Ischemic stroke patients demonstrate increased carotid plaque microvasculature compared to (Ocular) transient ischemic attack patients Cerebrovasc Dis 44: 297-303, 2017.

21. Zhang Q, An R, Tian X, Yang M, Li M, Lou J, Xu L and Dong $\mathrm{Z}: \beta$-Caryophyllene pretreatment alleviates focal cerebral ischemia-reperfusion injury by activating PI3K/Akt signaling pathway. Neurochem Res 42: 1459-1469, 2017.

22. Zhang X, Du Q, Yang Y, Wang J, Liu Y, Zhao Z, Zhu Y and Liu C: Salidroside alleviates ischemic brain injury in mice with ischemic stroke through regulating BDNK mediated PI3K/Akt pathway. Biochem Pharmacol 156: 99-108, 2018.

23. Yen TL, Chen RJ, Jayakumar T, Lu WJ, Hsieh CY, Hsu MJ, Yang $\mathrm{CH}$, Chang $\mathrm{CC}$, Lin YK, Lin $\mathrm{KH}$ and Sheu JR: Andrographolide stimulates p38 mitogen-activated protein kinase-nuclear factor erythroid-2-related factor 2-heme oxygenase 1 signaling in primary cerebral endothelial cells for definite protection against ischemic stroke in rats. Transl Res 170: 57-72, 2016.

24. Chern CM, Liou KT, Wang YH, Liao JF, Yen JC and Shen YC: Andrographolide inhibits PI3K/AKT-dependent NOX2 and iNOS expression protecting mice against hypoxia/ischemia-induced oxidative brain injury. Planta Med 77: 1669-1679, 2011.

25. Wada T and Penninger JM: Mitogen-activated protein kinases in apoptosis regulation. Oncogene 23: 2838-2849, 2004.

26. Yu ZH, Cai M, Xiang J, Zhang ZN, Zhang JS, Song XL, Zhang W, Bao J, Li WW and Cai DF: PI3K/Akt pathway contributes to neuroprotective effect of Tongxinluo against focal cerebral ischemia and reperfusion injury in rats. J Ethnopharmacol 181: 8-19, 2016.

27. Bao Y, Qin L, Kim E, Bhosle S, Guo H, Febbraio M, Haskew-Layton RE, Ratan R and Cho S: CD36 is involved in astrocyte activation and astroglial scar formation. J Cereb Blood Flow Metab 32: 1567-1577, 2012.

28. Freeman MR: Specification and morphogenesis of astrocytes. Science 330: 774-778, 2010.

29. Lanfranconi S, Locatelli F, Corti S, Candelise L, Comi GP, Baron PL, Strazzer S, Bresolin N and Bersano A: Growth factors in ischemic stroke. J Cell Mol Med 15: 1645-1687, 2011.

30. Erpolat S, Celik HT and Bozkurt B: Brain-derived neurotrophic factor is increased in serum levels of patients with symptomatic dermographism. Postepy Dermatol Alergol 34: 346-349, 2017.

31. Ya BL, Liu Q, Li HF, Cheng HJ, Yu T, Chen L, Wang Y, Yuan LL, Li WJ, Liu WY and Bai B: Uric acid protects against focal cerebral ischemia/reperfusion-induced oxidative stress via activating Nrf2 and regulating neurotrophic factor expression. Oxid Med Cell Longev 2018: 6069150, 2018.

32. Wang J, Ma W and Liu Y: Long non-coding RNA HULC promotes bladder cancer cells proliferation but inhibits apoptosis via regulation of ZIC2 and PI3K/AKT signaling pathway. Cancer Biomark 20: 425-434, 2017.

33. Franke TF, Kaplan DR and Cantley LC: PI3K: Downstream AKTion blocks apoptosis. Cell 88: 435-437, 1997.

34. Calleja V, Alcor D, Laguerre M,Park J,Vojnovic B,Hemmings BA, Downward J, Parker PJ and Larijani B: Intramolecular and intermolecular interactions of protein kinase B define its activation in vivo. PLoS Biol 5: e95, 2007.

This work is licensed under a Creative Commons Attribution-NonCommercial-NoDerivatives 4.0 International (CC BY-NC-ND 4.0) License. 